\title{
Work Storage in States of Apparent Negative Thermodynamic Temperature
}

\author{
Henning Struchtrup* \\ Mechanical Engineering, University of Victoria, Victoria, British Columbia V8W 2Y2, Canada
}

(Received 19 December 2017; revised manuscript received 27 April 2018; published 21 June 2018)

\begin{abstract}
Inverted quantum states provide a challenge to classical thermodynamics, since they appear to contradict the classical formulation of the second law of thermodynamics. Ramsey interpreted these states as stable equilibrium states of negative thermodynamic temperature, and added a provision to allow these states to the Kelvin-Planck statement of the second law [N. F. Ramsey, Phys. Rev. 103, 20 (1956)]. Since then, Ramsey's interpretation has prevailed in the literature. Here, we present an alternative option to accommodate inverted states within thermodynamics, which strictly enforces the original Kelvin-Planck statement of the second law, and reconciles inverted states and the second law by interpreting the former as unstable states, for which no temperature — positive or negative — can be defined. Specifically, we recognize inverted quantum states as temperature-unstable states, for which all processes are in agreement with the original Kelvin-Planck statement of the second law, and positive thermodynamic temperatures in stable equilibrium states. These temperature-unstable states can only be created by work done to the system, which is stored as energy in the unstable states, and can be released as work again, just as in a battery or a spring.
\end{abstract}

DOI: $10.1103 /$ PhysRevLett.120.250602

States of apparent negative thermodynamic temperatures were first produced in 1951 [1] by forced inversion of quantum states in select systems, and to the present day still garner widespread attention, e.g., Refs. [2-6].

Recent attempts to repudiate negative temperatures led to a vivid discussion on the definition of equilibrium entropy in statistical mechanics (Boltzmann versus Gibbs entropy) [3-6]. The following discussion relies on the Boltzmann definition of entropy, which leads to apparent negative temperatures.

Positive temperatures guarantee stability of equilibrium states, and apparent negative temperatures indicate unstable states. Nevertheless, only a few authors discuss the stability of the inverted quantum states, or question whether these are stable equilibrium states.

In a 1954 book review [7], Giauque writes, "The author's discussion in this section, of a 'formal' case of 'negative temperature', based on non-equilibrium nuclear spin states is very unfortunate. It is the sort of thing with which newspaper reporters can, and probably will, do a great deal of damage. Temperature has no meaning when the states used to define it are not in equilibrium. Innumerable cases in ordinary chemical thermodynamics could be cited in which corresponding nonequilibrium systems exist."

In contrast, Ramsey, in 1956 argued that inverted quantum states require modification of the KelvinPlanck statement of the second law of thermodynamics, which in essence forbids that work can be obtained from a single system in stable thermodynamic equilibrium [8]. Throughout his treatment, Ramsey assumes that the observed states are stable equilibrium states, dismissing Giauque's statement as a misunderstanding.
Most subsequent authors considering negative thermodynamic temperature built on Ramsey's approach [2,5,6,8-10], implicitly assuming stable equilibrium states.

More recently, Romero-Rochin [11] showed that states of apparent negative temperature are unstable in the sense that the smallest bit of standard material—which can only have positive temperature-brought in contact with an arbitrarily large system of apparent negative temperature will force the latter to assume a positive temperature.

This makes it impossible to measure the temperature of systems in inverted states by conventional means, i.e., a thermometer made of an arbitrary material, since in the equilibration between inverted system and thermometer, the inverted system will fall back into a state of positive temperature. Hence, in the following, we shall speak of these states as temperature-unstable states.

Moreover, a large inverted system cannot be considered as a heat reservoir, which is defined as a system whose change in temperature is negligible when it exchanges heat with another system [12] and which passes through stable equilibrium states only [13].

Hoffmann [14] described inverted quantum systems as mixtures in nonequilibrium states.

It will be shown that the classical interpretation of thermodynamics provides a complete description of inverted quantum states as temperature-unstable states. The systems are trapped in their unstable state, but will return to stable equilibrium states if provided a means of work or heat exchange with their surroundings. As in mechanical systems, or in chemically unstable mixtures, work can be stored in, and retrieved from, the energy of the unstable states. 
Since thermodynamic temperature is only defined for stable equilibrium states [13], it is impossible to define a temperature for the inverted states, to which one cannot assign a (positive or negative) thermodynamic temperature. Indeed, when inverted quantum states are recognized as temperature-unstable states, there is no need for Ramsey's modification of the second law of thermodynamics.

The second law of thermodynamics describes the evolution of thermodynamic systems towards stable equilibrium states $[13,15]$. In the language of irreversible thermodynamics, the deviation from a stable equilibrium state provides a thermodynamic driving force for a process towards stable equilibrium [16] (also see Supplemental Material [17], Sec. I).

Work can be produced from a process towards stable thermodynamic equilibrium. Indeed, all work producing thermodynamic engines are driven by thermodynamic forces. For example, the driving force for a heat engine is the temperature difference between the two thermal reservoirs with which the engine interacts. Heat will flow from the hotter to the colder reservoir in an attempt to equilibrate, and a heat engine converts some of the heat flowing into work $[13,15,17]$.

Work can also be produced from mechanical energy stored in a system, where the work is equal to the energy difference to the state of minimum energy (ground state) of the system. Examples are a compressed spring that can do work until fully relaxed, or the swirling motion of a fluid, from which work can be produced until the fluid comes to rest. The driving force for these work producing processes is the deviation from the ground state.

The Kelvin-Planck statement of the second law of thermodynamics states that it is impossible to construct an engine that will work in a complete cycle and produce no effect except the raising of a weight (i.e., produce work) and the transfer of energy out of a system (e.g., a reservoir) in a stable equilibrium state [13] (for other, equivalent, fomulations see, e.g., Refs. $[8,12,15])$.

While it is often not explicitly stated, it is understood that the reservoir is (a) in a stable thermodynamical equilibrium state and (b) in its mechanical ground state.

Inverted quantum states are reached by sudden field inversion, and the analysis shows that a system in an inverted quantum state appears to have negative temperatures (see below, and also Ref. [17], Sec. III), and can produce work-in contradiction to the Kelvin-Planck statement. There are two possible approaches for resolution.

Stance I.-One might consider the Kelvin-Planck statement to be universally valid, and hence conclude that inverted quantum states are either unstable equilibrium states or akin to elevated mechanical states.

Stance II.-One might consider inverted states as stable equilibrium states, and hence must extend the KelvinPlanck statement to allow these special states $[8,12,13]$.

Ramsey chose stance II, by declaring the above formulation to be valid for reservoirs at positive temperatures, and adding the following (Kelvin-Planck-Ramsey statement): "It is impossible to construct an engine that will operate in a closed cycle and produce no effect other than (2) the rejection of heat into a negative-temperature reservoir with the corresponding work being done on the engine" [8]. Note that this statement allows the opposite, namely, the production of work.

Following stance I, we will argue that inverted quantum states of apparent negative temperature can be described within classical thermodynamics, if they are considered to not be in a stable equilibrium state. The subsequent discussion will show that inverted states can be described as temperature-unstable states that store the work required for their creation as energy, which can be converted into work again. With this, they are akin to chemically unstable states, or mechanical systems away from their ground state.

For instance, a mixture of hydrogen, oxygen, and water vapor behaves like an inert gas mixture for a wider range of temperatures and pressures. This mixture is in thermal and mechanical equilibrium (homogeneous temperature and pressure), but trapped in a chemically unstable state. Using membrane separation and a fuel cell, (electrical) work can be produced from the mixture (Ref. [17], Sec. II). This does not violate the original Kelvin-Planck statement, since the mixture is not in full-thermal, mechanical, and chemical—stable equilibrium.

The driving force for the process is the deviation from stable chemical equilibrium, the fuel cell produces work from the driving force, as the system moves towards stable equilibrium. In the inversion of the process, the fuel cell is replaced by an electrolyzer which consumes work to split water, moving the system further away from stable equilibrium.

With work consumed in the electrolyzer, and work produced by the fuel cell, work can be stored in, and retrieved from, the energy of the trapped chemical states.

Similarly, mechanical systems can be brought out of their ground state by doing work, e.g., compression of a spring, acceleration of a flywheel, or inversion of a rigid pendulum. Work can be retrieved when the system returns to the ground state.

In both cases, due to irreversible processes, the work done by the system in discharging is smaller than the work done to the system in charging (Ref. [17], Sec. II).

To proceed, we consider the simplest model possible, where the quantum systems have a single degree of freedom, the occupation number of quantum elements. There are no other parameters to describe the system, or all other parameters remain frozen. Bounded energy of quantum states and energy exchange between the quantum elements $[2,8]$ lead to a relation between system entropy $S$ and system energy $E$ given by a curve $S(E)$ which first increases to a maximum and then decreases (Fig. 1).

Since the system has no additional parameters, (reversible) work exchange described by a pair of conjugate 


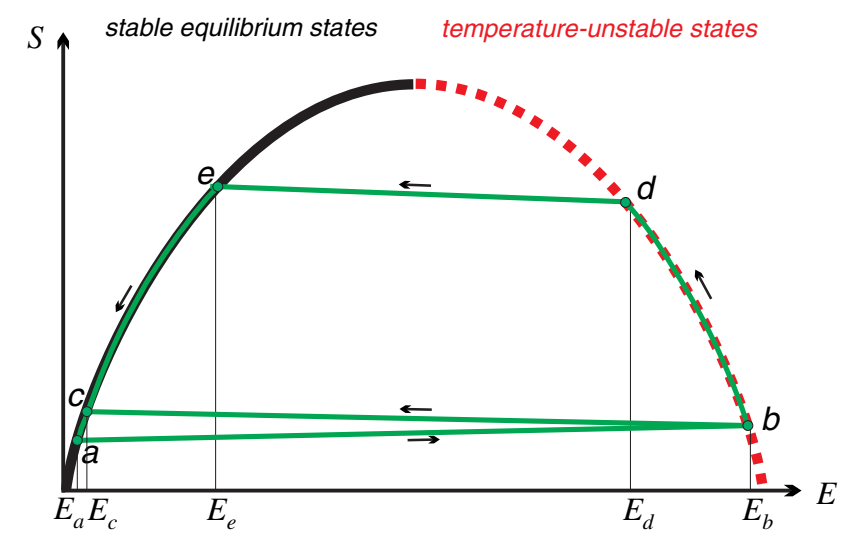

FIG. 1. Processes on and across the entropy-energy relation $S(E)$. States on the ascending part of the curve are stable equilibrium states (black), states on the descending part are temperature-unstable states (red dashes). Processes $a \rightarrow b \rightarrow c$ and $a \rightarrow b \rightarrow d \rightarrow e \rightarrow a$ are permitted irreversible processes; see discussion in the text.

variables, as in $\dot{W}=Y(d Z / d t)$ [12], is excluded. For the discussion on the grounds of thermodynamics, no deeper knowledge of the system and its manipulation, such as mechanisms for the work exchange, is required. The Supplemental Material (Ref. [17], Sec. III) uses a simple quantum model [14] to illustrate some aspects of these systems and the processes.

We only require the general form of the curve $S(E)$ and the first and second law of thermodynamics, which we write as [15] (see also Ref. [17], Sec. I)

$$
\frac{d E}{d t}=\dot{Q}-\dot{W}, \quad \frac{d S}{d t}-\frac{\dot{Q}}{T_{R}}=\dot{S}_{\text {gen }} \geq 0 .
$$

Here, $\dot{Q}$ is the heat transfer rate (positive for heat added to the system), $\dot{W}$ is the power (positive for work done by the system), $T_{R}$ is the temperature at which heat crosses the system boundary, and $\dot{S}_{\text {gen }}$ is the entropy generation rate, which is positive in nonequilibrium and vanishes in equilibrium.

With the relation $S(E)$, the two laws combine to

$$
\left(\frac{d S}{d E}-\frac{1}{T_{R}}\right) \dot{Q}-\frac{d S}{d E} \dot{W}=\dot{S}_{\text {gen }} \geq 0 .
$$

To clarify the meaning of $(d S / d E)$, we study two simple processes. For a pure heat transfer process between the system and a thermal reservoir at temperature $T_{R}$, there is no work, $\dot{W}=0$, and we have

$$
\left(\frac{d S}{d E}-\frac{1}{T_{R}}\right) \dot{Q}=\dot{S}_{\text {gen }} \geq 0 .
$$

Heat $\dot{Q}$ will flow until the entropy generation vanishes and the final equilibrium state, indicated by a subscript $E$, is reached, where $[11,15]$

$$
\left(\frac{d S}{d E}\right)_{\mid E}=\frac{1}{T_{R}}
$$

Temperature is defined such that it is equal between two systems in stable thermal equilibrium $[12,13,15]$; hence, in this final state, system temperature $T$ equals reservoir temperature $T_{R}$, which gives the definition of thermodynamic temperature of the system in stable equilibrium:

$$
\left(\frac{d S}{d E}\right)_{\mid E}=\frac{1}{T} .
$$

According to the Clausius statement of the second law $[12,13,15,17]$ heat must go from hot to cold by itself. Assuming the system is in stable equilbrium states during the process, Eq. (3) can be written as

$$
\left(\beta-\beta_{R}\right)(-\dot{Q})=\dot{S}_{\text {gen }} \geq 0,
$$

where $\beta=-(1 / T)$. If the system is hotter than the reservoir, the second law requires that the system loses heat, $\dot{Q}<0$; hence, $\beta>\beta_{R}$. The parameter $\beta$ provides a natural temperature scale from coldest, $\beta=-\infty$, to hottest, $\beta=+\infty$, so that negative temperatures $(\beta>0)$ refer to hotter states than positive temperatures $(\beta<0)$ [8]. However, following stance I, negative temperatures $(\beta>0)$ are excluded due to the original Kelvin-Planck statement, as is shown next.

For an adiabatic process, where $\dot{Q}=0$, the combined laws Eq. (2) reduce to

$$
-\frac{d S}{d E} \dot{W}=\dot{S}_{\text {gen }} \geq 0 .
$$

Work done by the system is positive. For the case that the system is in a stable thermodynamic equilibrium state, the original Kelvin-Planck statement forbids positive work, but allows us to supply work to the system, $\dot{W}=-|\dot{W}|<0$; hence,

$$
\left(\frac{d S}{d E}\right)_{\mid E}|\dot{W}|=\frac{1}{T}|\dot{W}|=\dot{S}_{\text {gen }} \geq 0 .
$$

This relation requires positive thermodynamic temperature:

$$
\left(\frac{d S}{d E}\right)_{\mid E}=\frac{1}{T}=-\beta \geq 0 .
$$

In summary, according to the second law interpretation of stance I, only states on the ascending branch of the $S(E)$ curve are stable equilibrium states, and these have positive temperatures.

Then, high energy states on the descending branch must be akin to unstable states, or elevated mechanical states, and we speak of temperature-unstable states. The second law (stance I) does not forbid that these produce work; with $\dot{W}=|\dot{W}|>0$, we have 


$$
-\left(\frac{d S}{d E}\right)|\dot{W}|=\dot{S}_{\text {gen }} \geq 0
$$

Work is produced in an irreversible process while the energy of the system decreases and its entropy increases, on the way towards stable equilibrium.

Ramsey [8] and others, following stance II, considered these states as stable, and defined the system temperature for all states through $(1 / T)=(d S / d E)$, which leads to negative temperatures for the descending branch, and requires Ramsey's addendum to the Kelvin-Planck statement.

We study storage of energy in inverted quantum states. No continuous process on the curve can connect the ascending and descending branches of the curve [10] (see also Ref. [17], Sec. III). Therefore, to produce a state of apparent negative temperature, one has to leave the curve, and "jump" from the stable branch to the unstable branch. This cannot be done by heating, and, to not have the quantum system fall back to thermal equilibrium with the reservoir, the system must be thermally detached, i.e., $\dot{Q}=0$.

For such a process, shown as curve $a \rightarrow b$ in Fig. 1, the first and second law are integrated over the process to give

$$
E_{b}-E_{a}=-W_{a b}>0, \quad S_{b}-S_{a}=S_{\mathrm{gen}}^{a b} \geq 0 .
$$

Here, $W_{a b}<0$ is the work required to change the energy from $E_{a}$ to $E_{b}$, and $S_{\text {gen }}^{a b}$ is the generation of entropy in this possibly irreversible process.

Such a state can be reached by state inversion [1]. A perfect inversion process is reversible [14], with $S_{b}=S_{a}$ and $S_{\text {gen }}^{a b}=0$. For an imperfect inversion, entropy is generated, $S_{b}>S_{a}$.

The work added to force the system into the unstable state $b$ can, at least in part, be recovered by allowing the system to return to the stable branch, in a reverse inversion process $b \rightarrow c$, for which the laws give

$$
E_{c}-E_{b}=-W_{b c}<0, \quad S_{c}-S_{b}=S_{\text {gen }}^{b c} \geq 0 .
$$

The system does work, $W_{b c}>0$, when returning from the unstable state $b$ to the stable state $c$, and the second law restricts the states that can possibly be reached to those with equal or larger entropy. Obtaining work from this process is not a violation of the second law, since state $b$ is not a stable equilibrium state, but a temperature-unstable state that stores energy equal to the work required for its creation. While the process $a \rightarrow b$ must be forced-work is required to bring the system out of stable equilibriumthe inverse process $b \rightarrow c$ is driven by the desire to reach stable equilibrium.

The difference between work for charge and discharge is the work loss,

$$
W_{\text {loss }}^{a b c}=\left|W_{a b}\right|-W_{b c}=E_{c}-E_{a} \geq 0,
$$

where $W_{\text {loss }}^{a b c}=0$ for the case of reversible charge and discharge $\left(E_{c}=E_{a}\right)$. Positive loss for the irreversible case can be read from Fig. 1, where the entropy generated is $S_{\text {gen }}^{a b c}=S_{c}-S_{a} \geq 0$.

Instead of returning from unstable state $b$ to the stable branch, one might run a process through the unstable states, on the descending branch of the curve $S(E)$ to state $d$. The quantum system is still detached from the heat bath; hence, the process is adiabatic. For the process $b \rightarrow d$ on the curve, the laws reduce to

$$
E_{d}-E_{b}=-W_{b d}<0, \quad S_{d}-S_{b}=S_{\mathrm{gen}}^{b d} \geq 0 .
$$

Entropy must grow, and the curve in Fig. 1 shows that energy will decrease. Accordingly, work $W_{b d}>0$ is done by the system, while the process is irreversible, with positive entropy generation $S_{\mathrm{gen}}^{b d}$. This process does not violate the second law (stance I): we observe a system in a temperature-unstable state in a process towards its stable equilibrium state. The work $W_{b d}$ obtained is part of the work $W_{a b}$ stored in the energy of the temperatureunstable state.

For a fuller picture, we study a closed process loop, with the following processes (see Fig. 1): $(a \rightarrow b)$ state inversion by work: $(b \rightarrow d)$ work done in an irreversible process; $(d \rightarrow e)$ work done in reverse state inversion; $(e \rightarrow a)$ return to initial state by cooling.

Stable state $e$ has higher energy, and higher temperature $T_{e}$, than the initial state $a$. More work is available from cooling the system by means of a heat engine, from $T_{e}$ to reservoir temperature $T_{a}$. Assuming a reversible engine, the first and second law show that one can obtain the work [15]

$$
W_{e a}=\left(E_{e}-E_{a}\right)-T_{a}\left(S_{e}-S_{a}\right)>0 .
$$

The overall work loss for the complete process is

$$
W_{\text {loss }}^{\text {abdea }}=\left|W_{a b}\right|-W_{b d}-W_{d e}-W_{e a}=T_{a}\left(S_{e}-S_{a}\right) \geq 0 .
$$

Hence, the overall work loss of charging and discharging the quantum system is the reservoir temperature $T_{a}$ times the overall entropy generation of the storage process, just as for a chemically unstable mixture (see Ref. [17], Sec. II).

Next, we discuss heat transfer involving inverted quantum systems. As stated above, systems in inverted quantum states will fall back to stable equilibrium states, and positive temperatures, when they come in thermal contact with standard systems $(T>0)$ of arbitrary size [11]. In this case, some of the work stored in the energy of temperatureunstable states is dissipated by irreversible processes. 
One might also bring two systems (of the same type) that allow inverted states into contact. In this case, the interaction between the quantum elements will lead to a mixing process [14], the redistribution of energy between quantum elements, where, dependent on the size of the two systems and their initial states, the final state can be on any branch of the curve. Total energy is conserved, and due to the convexity of the curve $S(E)$, entropy increases; that is, the process is irreversible (see Ref. [17], Sec. III).

Indeed, the only way to move a system in a temperatureunstable state (say, state $b$ in Fig. 1) to higher energies on the curve (i.e., without a detour through stable states) is through contact with an inverted system $x$ at higher specific energy $\left(E_{x} / n_{x}\right)>\left(E_{b} / n_{b}\right)\left(n_{x}, n_{b}\right.$ are the mole numbers of the two systems). Both systems store energy equal to the work required for their creation, and after the interaction some of this energy is lost to entropy generation, and cannot be recovered as work anymore.

This process of energy transfer between systems of invertible quantum states can be described as heat transfer between states of apparent negative temperature, from hot to cold. Hence, in a world of only invertible quantum systems, $(d S / d E)<0$ might be considered as temperature to describe heat transfer.

However, one must not mistake $(d S / d E)<0$ for a measurable temperature in the classical sense, since measurement requires stable thermal equilibrium between thermometer and system, without any restriction on the thermometer material. Similarly, a large system of inverted quantum states might be considered as a heat bath for a small system made of the same material, but not for a small system of any other material. Indeed, contact with the latter, or a thermometer, will have the inverted states fall back into stable equilibrium states at $(d S / d E)>0$ [11].

We have cited only a few contributions from the literature which followed Ramsey's modification of the second law (stance II), and evaluate, e.g., heat engines operating between reservoirs of positive and negative thermodynamic temperatures [9]. This requires heat transfer into, and out of, systems in states of apparent negative temperatures, which can only occur between systems made from the same material. Carnot efficiencies at [5] or above [4] unity might occur since reservoirs in temperatureunstable states can release the work that was required for their generation. No general conclusions on Carnot and other engines-made from arbitrary materials-involving negative temperatures and heat transfer are possible.

Inverted quantum states are special states [13], indeed, since they can be understood as being not in stable equilibrium. They have a single degree of freedom, which is frozen as long as a system remains isolated. Any contact with the surroundings, either by heat transfer or by work, allows the system to approach stable states. One can determine their energy-entropy relation, but, since temperature is defined for stable equilibrium states, one cannot assign a temperature to these states. The work required for their creation is stored as energy of the special state, just like in a battery or in a mechanical spring, and can be recovered.

There is no contradiction to classical thermodynamics (stance I) when inverted quantum states of apparent negative temperature are recognized as nonstable. Negative thermodynamic temperatures of stable equilibrium states violate the second law of thermodynamics, and should not be used.

This research was triggered by a discussion on Research Gate [19], and I would like to thank all participants, in particular Professor Oliver Penrose (Edinburgh), for the helpful discussion. Moreover, I am grateful for the anonymous reviewers' constructive criticism, which helped me further to shape a coherent picture of the relation between apparent negative temperatures and classical thermodynamics. Support from the National Science and Engineering Research Council of Canada (NSERC) is gratefully acknowledged.

*struchtr@uvic.ca

[1] E. M. Purcell and V. Pound, Phys. Rev. 81, 279 (1951).

[2] S. Braun, J. P. Ronzheimer, M. Schreiber, S. S. Hodgman, T. Rom, I. Bloch, and U. Schneider, Science 339, 52 (2013).

[3] J. Dunkel and S. Hilbert, Nat. Phys. 10, 67 (2014); I. M. Solokov, Nat. Phys. 10, 7 (2014).

[4] D. Frenkel and P. B. Warren, Am. J. Phys. 83, 163 (2015).

[5] E. Abraham and O. Penrose, Phys. Rev. E 95, 012125 (2017).

[6] R. H. Swendsen, Entropy 19, 603 (2017).

[7] W. F. Giauque, J. Am. Chem. Soc. 76, 5577 (1954).

[8] N. F. Ramsey, Phys. Rev. 103, 20 (1956).

[9] P. T. Landsberg, J. Phys. A 10, 1773 (1977).

[10] A.-M. Tremblay, Am. J. Phys. 44, 994 (1976).

[11] V. Romero-Rochin, Phys. Rev. E 88, 022144 (2013).

[12] J. Kestin, A Course in Thermodynamics (Blaisdell Publishing Company, Waltham MA, 1966).

[13] E. P. Gyftopoulos and G.P. Beretta, ThermodynamicsFoundations and Applications (Dover Publications, Mineola, NY, 2005).

[14] H.-J. Hoffmann, Materialwiss. Werkstofftech. 46, 765 (2015).

[15] H. Struchtrup, Thermodynamics and Energy Conversion (Springer, Heidelberg, 2014), DOI: 10.1007/978-3-66243715-5.

[16] S. R. de Groot and P. Mazur, Non-Equilibrium Thermodynamics (North-Holland Publishing Company, Amsterdam, 1962).

[17] See Supplemental Material at http://link.aps.org/ supplemental/10.1103/PhysRevLett.120.250602, which reviews elements of thermodynamics to provide additional background for the discussion in the main Letter, and which includes Ref. [18].

[18] H. Struchtrup, Macroscopic Transport Equations for Rarefied Gas Flows, Interaction of Mechanics and Mathematics Series (Springer, Heidelberg, 2005), DOI: 10.1007/3-54032386-4.

[19] https://tinyurl.com/y7e312s8. 